\title{
Improved Rooting and Acclimatization of Micropropagated Hazelnut Shoots
}

\author{
Mehmet Nuri Nas ${ }^{1}$ \\ Kahramanmaras Sutcu Imam University Faculty of Agriculture, Department \\ of Horticulture, 46060 Kahramanmaras, Turkey
}

Paul E. Read

Department of Agronomy and Horticulture, University of Nebraska-Lincoln Institute of Agronomy and Natural Resources, 377 Plant Sciences, Lincoln, NE 68583-0724

Additional index words. acclimatization, copper, $\mathrm{Cu}$, Corylus, hardening, myo-inositol, rooting, ex vitro survival

\begin{abstract}
Microshoots of four hazelnut genotypes grown in vitro on Nas and Read medium (NRM) containing various combinations of $\mathrm{CuSO}_{4} \cdot 5 \mathrm{H}_{2} \mathrm{O}$ and myo-inositol were successfully rooted and acclimatized ex vitro without any need of in vitro hardening treatments. Dipping of shoot bases in 1000 ppm indole-3-butyric acid (IBA) solution for 5 or 10 seconds followed by placement of shoots in plant growth regulator free NRM gave rise to formation of roots as early as 8 days. Shoots treated for 5 and 10 seconds rooted similarly, and depending on genotype, $88 \%$ to $98 \%$ rooting was observed within 15 days after treatment with IBA. Ex vitro survival of shoots three months after in vitro-root induction was $73 \%$ when shoots were treated with IBA for 5 seconds and $66 \%$ when shoots were treated for 10 seconds. The highest ex vitro survival rate $(97 \%) 3$ months after root induction was observed when shoots were treated with IBA solution for 10 seconds, and then cultured directly in peat pellets. Shoots developed good roots, and grew up to $70 \mathrm{~cm}$ in height 3 months after root induction. The potential use of rooting and acclimatization protocol for commercial micropropagation of hazelnut is presented.
\end{abstract}

Hazelnut is one of the most important nut crops in the world (Fallico et al., 2003; Ozdemir and Akinci, 2004). Many countries have promoted its production, and breeding programs have emphasized the development of elite lines of improved production to meet domestic demand and avoid high-priced imports (Mehlenbacher, 2001). However, increased demand for hazelnut products can only be met by a rapid plant propagation scheme and efficient distribution of elite lines. Traditional hazelnut propagation methods such as layering are time-consuming, laborious and unsatisfactory for commercial propagation. Micropropagation has been considered as an alternative to traditional hazelnut propagation techniques (Diaz-Sala et al., 1990; Nas and Read, 2004; Yu and Reed, 1995).

Corylus species are viewed as recalcitrant among tissue culturist. High rate of microbial contamination, oxidative browning of explants, low proliferation rate, insufficient shoot elongation and hyperhydricity have been reported to be the factors limiting the success of micropropagation (Anderson, 1983; Diaz-Sala et al., 1994; Nas and Read, 2001; Yu and Reed, 1995). As a part of developing a hazelnut micropropagation protocol, Nas and Read (2004) developed a new culture medium [Nas and Read medium (NRM)]. Using NRM

Received for publication 9 Dec. 2003. Accepted for publication 13 Apr. 2004. A contribution of the University of Nebraska Agricultural Research Division, Lincoln. Journal series 13529.

${ }^{1}$ Corresponding author; e-mail mnurinas@ksu. edu.tr. it was estimated that 2.3 and 8.7 million shoots could be obtained in 1 year (nine subcultures) growing a single-bud explant from a mature hazelnut plant, even if $20 \%$ shoots were lost at each subculture. Even with these high proliferation rates, Nas et al. (2004) reported that there was no evidence of genetic changes among shoots subjected to long-term repeated subculture for over 6 years when preexisting meristems (axillary buds and shoot tips $\leq 1.0$ $\mathrm{cm}$ ) were used as explants.

The culture medium has a great influence on the quality (Nas and Read, 2001), anatomy and acclimatization of microshoots (Ziv, 1991). While high proliferation rates and genetic integrity of plants are important, the ultimate success of a commercial micropropagation procedure depends largely on successful rooting, acclimatization, high survival rates and rapid plant growth ex vitro. Because of cultureinduced phenotype (Bhojwani and Razdan, 1996; Ziv, 1995), rooting and acclimatization of in vitro grown (especially woody) plants remain as challenging problems. To improve shoot elongation and rooting of microshoots, Maene and Debergh (1985) suggested addition of liquid medium to established stage II cultures growing on solid medium for several weeks. Many researchers have pointed out the importance of ex vitro rooting as it offers many advantages over in vitro rooting (Rogers and Smith, 1992). Other researchers have suggested in vitro hardening of plants (Dami and Hughes, 1997; Deng and Donnelly, 1993; Murali and Duncan, 1995; Smith et al., 1991; Ziv and Ariel, 1991). The objectives of this study were to explore rooting capacity, accli- matization and ex vitro growth performance of micropropagated hazelnut shoots cultured on NRM with various combinations of myoinositol and $\mathrm{Cu}$.

\section{Materials and Methods}

Influence of Copper sulfate and Myo-inositol concentration on shoot elongation and development. Culture medium, propagation method and culture conditions were outlined by Nas and Read (2004). For propagation (stage II) axillary buds were cultured in disposable clear plastic sundae cups [bowl: DSD8X - lid: LD8-58 (Sweetheart Cup Co., Md.] containing $80 \mathrm{ml} \mathrm{NRM}$ for 45 -day culture periods. Microshoots (1 to $4 \mathrm{~cm}$ ) of hybrid hazelnut [genotypes E-093-S, E-295-S, G-029-N (8to to 10-year-old stock plants), and S-182 (2-year-old stock plant)] grown in NRM supplemented with $22.2 \mu \mathrm{MBA}, 0.049 \mu \mathrm{M} \mathrm{IBA}$, $30 \mathrm{~g} \cdot \mathrm{L}^{-1}$ sucrose, and gelled with $6 \mathrm{~g} \cdot \mathrm{L}^{-1}$ agar (Sigma, A-1296) were used as experimental material. Modifications of the copper sulfate and myo-inositol concentrations tested were $0.025+100(\mathrm{C} 1 \mathrm{M} 1), 1.28+200(\mathrm{C} 2 \mathrm{M} 2), 2.55$ +400 (C3M3), $5.1+800$ (C4M4) amount in $\mathrm{mg} \cdot \mathrm{L}^{-1}$ of $\mathrm{CuSO}_{4} \cdot 5 \mathrm{H}_{2} \mathrm{O}$ and myo-inositol, respectively, to elucidate their effectiveness in stimulating subsequent rooting.

Rooting and acclimatization ofmicroshoots. The efficacy of in vitro versus ex vitro rooting and acclimatization of hazelnut microshoots was examined. For in vitro rooting, the base of microshoots obtained from NRM (stage II) containing various levels of $\mathrm{CuSO} \cdot 5 \mathrm{H}_{2} \mathrm{O}$ and myo-inositol were dipped in a $1000 \mathrm{ppm}$ IBA [dissolved in $90 \%$ ethanol then brought to final concentration of $20 \%$ ethanol $80 \%$ deinozed water $(\mathrm{v} / \mathrm{v})$ ] solution for 5 or $10 \mathrm{~s}$. IBA-treated shoots of each genotype were transferred to clear plastic sundae cups [DSD8X-LD8-58, (Sweetheart Cup Co.) containing $80 \mathrm{~mL}$ plant growth regulator (PGR)-free NRM (C1M1 level of Copper sulfate and myo-inositol ). Replicate vessels with four to six shoots were arranged in a completely randomized design with each genotype having two replicates (sundae cups) at each level of treatment (a total of 8 replicates). Rooted shoots were transplanted to nonsterile water-saturated peat plugs (No. 9) (Jiffy Products) and placed in sundae cups containing a small amount of water 8 to $15 \mathrm{~d}$ after treatment. All shoots transplanted into peat plugs were shifted to the greenhouse ( 22 to $26 \pm 3{ }^{\circ} \mathrm{C}$, and uncontrolled relative humidity) and placed on a bench shaded with cheesecloth to provide a 125 to $145 \mu \mathrm{mol} \cdot \mathrm{s}^{-1} \cdot \mathrm{m}^{-2}$ light intensity. Day length was extended to $16 \mathrm{~h}$ by providing 2 to $3 \mathrm{~h}$ of light at $40 \mu \mathrm{mol} \cdot \mathrm{s}^{-1} \cdot \mathrm{m}^{-2}$ using 400-W metal halide lamps. Rooted shoots were transplanted into pots containing a mixture of $60 \%$ peat and $40 \%$ perlite either when roots grew through the walls of the peat plugs or seven days after shoots were placed into peat plugs. Shoots transplanted to pots were covered with clear plastic sundae cup lids (LD8-58, (Sweetheart Cup Co.) for another week. At the end of the fourth week all sundae cup lids and the cheesecloth were removed and plants were left to grow under the standard greenhouse 
conditions (300 to $420 \mu \mathrm{mol} \cdot \mathrm{s}^{-1} \cdot \mathrm{m}^{-2}$ daylight, 13- to15-h photoperiod).

For ex vitro rooting, the base of microshoots (10 to 16) of each genotype obtained from NRM (stage II) containing various levels of $\mathrm{CuSO}_{4}$ • $5 \mathrm{H}_{2} \mathrm{O}$ and myo-inositol were dipped in a 1000 $\mathrm{ppm}$ IBA solution for $10 \mathrm{~s}$ before transfer to nonsterile water-saturated Jiffy peat plugs (No. 9) in sundae cups containing a small amount of water. All sundae cups (shoots) were placed on a bench shaded with the cheesecloth in the greenhouse as described for acclimatization of in vitro rooted shoots. However, shoots were transplanted to pots when roots were visible through the walls of the peat plugs or $21 \mathrm{~d}$ after application of IBA. The potting mixture was identical to that described previously. At the end of the fourth week all sundae cup lids and the cheesecloth were removed and plants grown under the greenhouse conditions that described above.

Data recorded and statistical analysis. The effects of the culture medium (level of $\mathrm{Cu}+$ myo-inositol) and the exposure time of shoots to 1000 ppm IBA solution on the percentage of shoots with root (in vitro) were analyzed using the PROC GLM (SAS, 2001) procedure with significance difference at $P=$ 0.05 level. To provide a normal distribution, percent rooting and surviving data were subjected to ARSIN [SQRT(X)] transformation. The parameters analyzed were days after application of IBA required for rooting (DR) of one or more shoots in one experimental unit, days required for rooting of $50 \%$ (R50\%) of shoots, and final per cent rooting (FR\%) of shoots $15 \mathrm{~d}$ after application of IBA. The effects of rooting methods (in vitro vs. ex vitro) on the survival and growth rate of shoots in the greenhouse were recorded three months after the application of IBA. Separation of treatment means was done by Bonferroni $t$ test if $\mathrm{F}$ test was significant. Unless mentioned otherwise, all in vitro and greenhouse experiments were conducted twice.

\section{Results and Discussion}

The effects of genotype, medium, exposure time of shoots to IBA solution and two-way interactions on the start of rooting (DR; $P=$ 0.28 ) or on the number of days for $50 \%$ rooting of shoots ( $\mathrm{R} 50 \% ; P=0.43)$ were insignificant. The effect of genotype ( $P=0.005)$ only on the final percent rooting of shoots (FR\%) was significant. However, the effects of medium $(\mathrm{P}$ $=0.48)$, the exposure time of shoots to IBA solution $(P=0.41)$ and the effect of two-way interactions (genotype $\times$ medium: $P=0.21$; genotype $\times$ IBA: $P=0.94$; medium $\times$ IBA: $P$ $=0.36$ ) on FR\% of shoots were insignificant.

Regardless of genotype, the culture medium (level of $\mathrm{Cu}+$ myo-inositol) or the exposure time of shoots to IBA solution, shoots formed roots as early as 8.2 to $8.6 \mathrm{~d}$ and $>50 \%$ of shoots rooted in 8.2 to $9.5 \mathrm{~d}$ after they were subjected to IBA solution. Shoots could form as many as 12 roots in $15 \mathrm{~d}$ (data not shown). Rooting of micropropagated hazelnut shoots has frequently been achieved by dipping the base of shoots in an IBA solution (1000 to

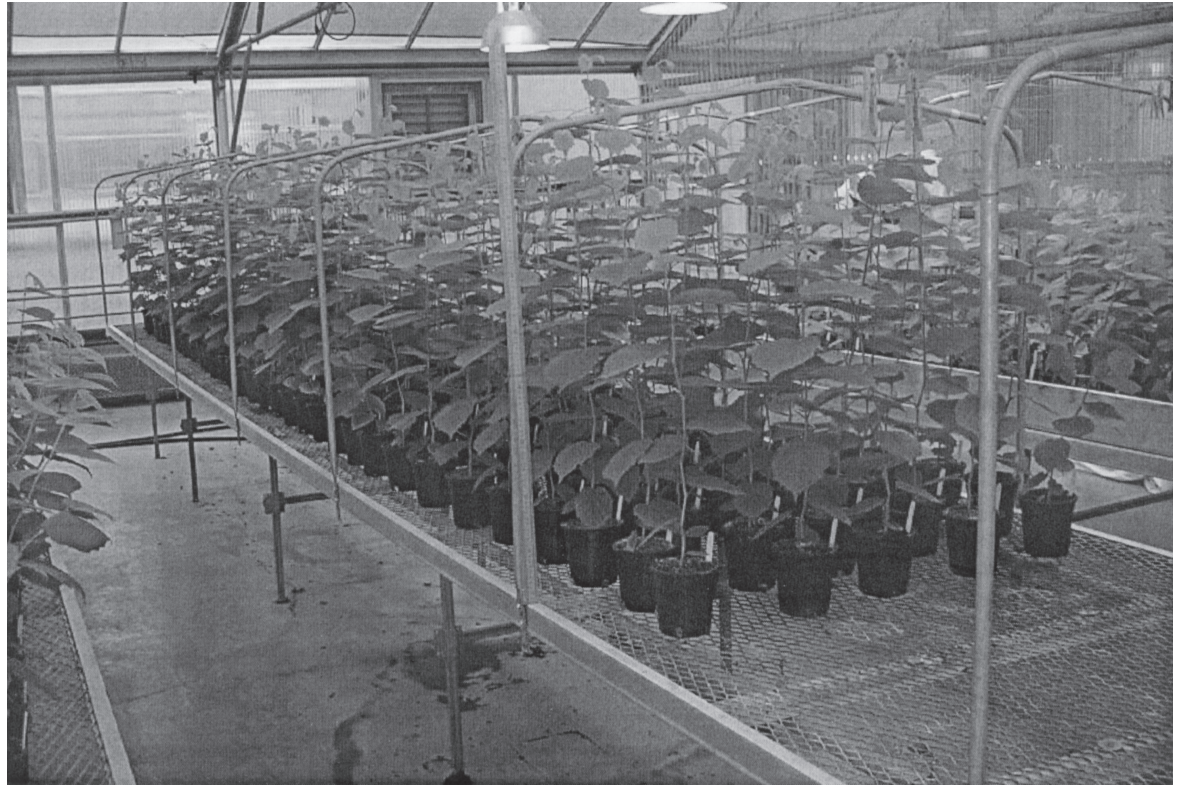

$1500 \mathrm{ppm}$ ) for 10 to $60 \mathrm{~s}$, and then culturing shoots in half-strength culture medium for 3 to 4 weeks (Diaz-Sala et al., 1990; Diaz-Sala et al., 1994; Rodriguez et al., 1988; Yu and Reed, 1995). Our results indicate that micropropagated hazelnut shoots could easily be rooted on full strength NRM in 2 weeks. Thus, rooting of microshoots is not a likely shortcoming of hazelnut micropropagation.

The combined mean FR\% for shoots of the four genotypes was $98 \%$ when the culture medium was $\mathrm{C} 1 \mathrm{M} 1,97 \%$ when the culture medium was $\mathrm{C} 2 \mathrm{M} 2,95 \%$ when the culture medium was $\mathrm{C} 3 \mathrm{M} 3$, and $93 \%$ when the culture medium was C4M4, respectively, and they were not significantly different from one another. The combined mean FR\% for shoots of the four genotypes treated with $1000 \mathrm{ppm}$ IBA solution for 5 and $10 \mathrm{~s}$ and cultured on PGR-free medium for $15 \mathrm{~d}$ were $97 \%$ and $95 \%$ and they were not significantly different from one another (data not shown).

The use of higher amounts of myo-inositol (osmoticum) in the culture medium is somewhat similar to hardening of plants in vitro. It has been suggested that the cost and ex vitro hardening period could be reduced if plants are hardened in vitro (Crane and Hughes, 1990; Dami and Hughes, 1997; Deng and Donnelly, 1993; Smith et al., 1991; Ziv, 1991). However, in vitro hardening methods could be detrimental for plant growth (Murali and Duncan, 1995; Ziv and Ariel, 1991). Although not significant, the combined FR\% of four genotypes observed on higher levels of $\mathrm{CuSO}_{4} \cdot 5 \mathrm{H}_{2} \mathrm{O}$ and myo-inositol was diminished also indicate that in vitro hardening may not always be beneficial.

The mean FR\% of genotype E-093-S (88\%) was lower compared to FR\% of E-295-S (98\%), G-029-N (98\%), and S-182 (98\%). Unlike other genotypes, E-093-S grew faster and it developed longer shoots at all levels of copper and myo-inositol (Nas and Read, 2004). The fast growing shoots of E-093-S were probably harder and they became relatively more difficult to root in $15 \mathrm{~d}$. The rooting abilities of the three genotypes used in this study (E-295S, G-029-N and S-182) on PGR-free woody

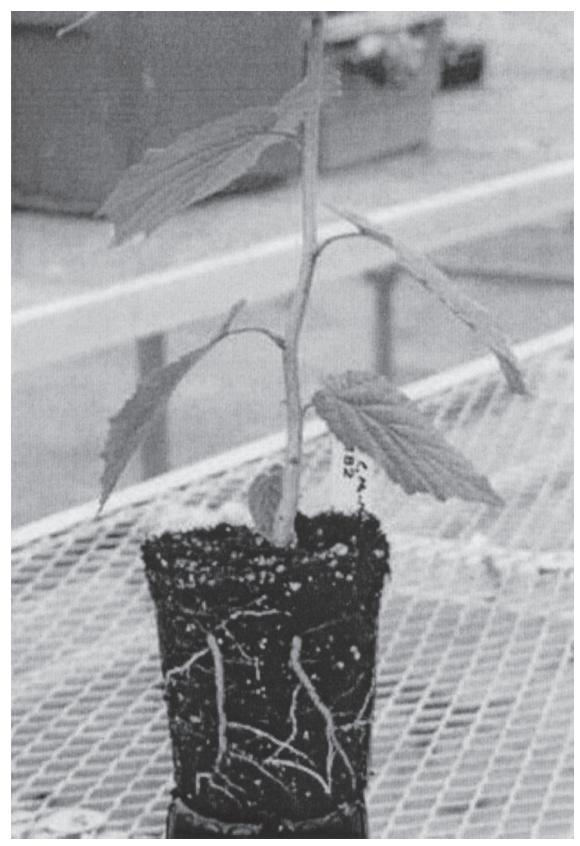

Fig. 1. Growth of micropropagated hybrid hazelnut shoots ex vitro. (top) Plants (in vitro and ex vitrorooted) grown in the greenhouse for $100 \mathrm{~d}$ after microshoots were treated with IBA solution (notice 1-m ruler). (bottom) Root structure of an ex vitrorooted shoot (G-029-N) grown in the greenhouse 3 months after microshoots were treated with IBA solution (pot removed for photo).

plant medium were reported previously (Nas and Read, 2003). Explants of E-295-S and G$029-\mathrm{N}$ were from older trees but they had been in culture for a longer time than that of S-182 and displayed greater rooting ability than the later. The rooting rates of microshoots were $100 \%, 78 \%$, and $26 \%$ for E-295-S, G-029-N, and S-182, respectively (Nas and Read 2003). Our new results obtained on PGR-free NRM indicate that rooting ability of microshoots of E-295-S (98\%) remained almost unchanged while those of G-029-N (98\%) and S-182 $(98 \%)$ have increased dramatically.

Although rooting rate of micropropagated plants from adult tissues is usually lower than 
that of juvenile tissues (Dhawan and Bhojwani, 1987; Rodriguez et al., 1988), we suggest that rooting of micropropagated shoots of mature origin can be improved simply by increasing the number of subculture cycles which is a prerequisite for mass propagation of many woody species. Diaz-Sala et al. (1994) also reported that repeated subculturing enhanced rooting of mature origin hazelnut shoots propagated in vitro.

Three months after in vitro root induction, ex vitro survival of shoots subjected to IBA solution for $5 \mathrm{~s}(66 \%)$ and $10 \mathrm{~s}(73 \%)$ were similar and were significantly $(P=0.005)$ lower than that of shoots $(97 \%)$ treated with IBA solution for $10 \mathrm{~s}$ and rooted ex vitro in Jiffy peat plugs. The lower survival rate observed when shoots were rooted in vitro could be explained by possible root damage during transplantation or poor vascular connections (Grout and Aston, 1977).

Three months after root induction, the effect of genotype on shoot height was the only significant factor $(P=0.005)$. The effect of culture medium $(P=0.14)$ or the effect of exposure time of shoots to IBA $(P=0.38)$ on shoot height were insignificant. Only $3.5 \%$ of plants that survived ex vitro were not able to develop shoots longer than $20 \mathrm{~cm}$ while some other shoots reached up to $70 \mathrm{~cm}$ in height (Fig. 1 , top). The mean shoot lengths were 38,44 , 48 , and $50 \mathrm{~cm}$ for shoots of genotypes E-093-S, E-295-S, G-029-N, and S-182, respectively. Plants grown in the greenhouse apparently developed good root systems that allowed plants to grow vigorously (Fig. 1, bottom).

Most studies involving rooting and ex vitro survival rate of micropropagated hazelnut have been terminated in 1 month ( $\mathrm{Yu}$ and Reed, 1995; Anderson, 1983) and no details of plant growth were reported. For commercial micropropagation ex vitro plant growth is also important. The shoot lengths $(38$ to $50 \mathrm{~cm}$ ) that we observed in the greenhouse three months after plants were taken from culture indicate that our rooting and acclimatization protocol can successfully be used for commercial propagation of hazelnut. Since ex vitro rooting and acclimatization gave the highest survival rate, and it is less time consuming than in vitro rooting, we suggest the preference of ex vitro rooting and acclimatization for future work.

\section{Literature Cited}

Anderson, W.C. 1983. Micropropagation of filberts, Corylus avellana L. Intl. Plant Prop. Soc. Comb. Proc. 33:132-137.

Bhojwani, S.S. and M.K. Razdan. 1996. Plant tissue culture: Theory and practice, p. 483-536. Revised ed. Elsevier, Amsterdam.

Crane, J. and H. Hughes. 1990. Medium overlays for improved hardening ofmicropropagated potatoes. HortScience 25:794-795.

Dami, I. and G. Hughes. 1997. Effects of PEGinduced water stress on in vitro hardening of 'Valiant' grape. Plant Cell Tiss. Org. Cult. 47:97-101.

Deng, R. and D.J. Donnelly. 1993. In vitro hardening of red raspberry by $\mathrm{CO}_{2}$ enrichment and reduced medium sucrose concentration. HortScience 28:1048-1051.

Dhawan, V. and S.S. Bhojwani. 1987. Hardening in vitro and morpho-physiological changes in the leaves during acclimatization of micropropagated plants of Leucaena leucocephala (Lam) de wit. Plant Sci. 53: 65-72.

Diaz-Sala C., M. Rey, and R. Rodriguez. 1990. In vitro establishment of cycloclonal chain from nodal segments and apical buds of adult hazel (Corylus avellana $\mathrm{L}$ ). Plant Cell Tiss. Org. Cult. 23:151-157.

Diaz-Sala C., M. Rey, and R. Rodriguez. 1994. Temporary modification of adult filbert proliferation capacity by sequential subcultures: Intensive pruning as a pre-treatment for in vitro reinvigoration. J. Hort. Sci. 69:673-678.

Fallico, B.,E. Arena, and M.Zappala. 2003. Roasting of hazelnuts. Role of oil in colour development and hydroxymethylfurfural formation. Food Chem. 81:569-573.

Grout, B.W.W. and M.J. Aston. 1977. Transplanting of cauliflower plants regenerated from meristem culture. I. Water loss and water transfer related to changes in leaf wax and to xylem regeneration. Hort. Res. 17:1-7.

Maene, L. and P. Debergh. 1985. Liquid medium addition to established tissue cultures to improve elongation and rooting in vivo. Plant Cell Tiss.
Org. Cult. 5:23-33.

Mehlenbacher, S.A. (ed). 2001. $5^{\text {th }}$ Int. Congr. on hazelnut. Acta Hort. 556.

Murali, T.P. and E.J. Duncan. 1995. The effects of in vitro hardening using triazoles on growth and acclimatization of banana. Sci. Hort. 64:243-251.

Nas, M.N. and P.E. Read. 2001. Micropropagation of hybrid hazelnut: Medium composition, physical state and iron source affect shoot morphogenesis, multiplication and explant vitality. $5^{\text {th }}$ Intl. Congr. on hazelnut. Acta Hort. 556:251-258.

Nas, M.N. and P.E. Read. 2003. Ex vitro survival of hybrid hazelnut shoots produced in vitro. $1^{\text {st }}$ Intl. Symp. on acclimatization and establishment of micropropagated plants. Acta Hort. 616:215-219.

Nas, M.N. and P.E. Read. 2004. A hypothesis for the development of a defined tissue culture medium of higher plants and micropropagation of hazelnuts. Sci. Hort. 101:189-200.

Nas, M.N., N. Mutlu, and P.E. Read. 2004. Randomly amplified polymorphic DNA(RAPD) analysis of long-term cultured hybrid hazelnut. HortScience 39:1079-1082.

Ozdemir, F. and I. Akinci. 2004. Physical and nutritional properties of four major commercial Turkish hazelnut varieties. J. Food Eng. 63:341-347.

Rodriguez, R., C. Diaz-Sala, and G. Ancora. 1988. Sequential cultures of explants taken from adult Corylus avellana L. Acta. Hort. 227:460-463.

Rogers, R.B. and M.A.L. Smith. 1992. Consequences of in vitro and ex vitro root initiation for miniature rose production. J. Hort. Sci. 67:535-540.

Smith, E.F., A.V. Roberts, and J. Mottley. 1991. The preparation in vitro of Chrysanthemum for transplantation to soil. III. The effect of eleven growth retardants on wilting. Plant Cell Tiss. Org. Cult. 27:309-317.

Yu, X.L. and B.M. Reed. 1995. A micropropagation system for hazelnut (Corylus species). HortScience 30:120-123.

Ziv, M. and T. Ariel. 1991. Bud proliferation and plant regeneration in liquid-cultured philodendron treated with ancymidol and paclobutrazol. J. Plant Growth Regul. 10:53-57.

Ziv, M. 1991. The use of growth retardants for the regulation and acclimatization of in vitro plants. Progress in plant growth regulation, p. 809-817. Proc. $14^{\text {th }}$ Intl. Conf. on plant growth substances, Amsterdam. 